\title{
Research Progresses in the Inhibitory Effect of Bone Mesenchymal Stem Cell-Derived Exosome on Blood-Brain Barrier Disruption following Intracerebral Hemorrhage in Rats
}

\author{
Gang Yang ${ }^{* \#, ~ Z h a n w e i ~ R u a n ~}{ }^{2 *}$, Chenbing Wang1, Chao Gu${ }^{1}$, Junjie Lv ${ }^{1}$, Shaojun Yang1, \\ Lulu Weng1, Feng Ding1, Long Ai1, Donghai Yuan'1, Fei Chen'1, Jiangli Chen', Gaofeng Shao1 \\ ${ }^{1}$ Department of Neurosurgery, Zhuji Affiliated Hospital of Shaoxing University, Shaoxing, China \\ ${ }^{2}$ Department of Emergency, The Third Affiliated Hospital, Wenzhou Medical University, Wenzhou, China \\ Email: "tekeyang@sina.com
}

How to cite this paper: Yang, G., Ruan, Z.W., Wang, C.B., Gu, C., Lv, J.J., Yang, S.J., Weng, L.L., Ding, F., Ai, L., Yuan, D.H., Chen, F., Chen, J.L. and Shao, G.F. (2021) Research Progresses in the Inhibitory Effect of Bone Mesenchymal Stem CellDerived Exosome on Blood-Brain Barrier Disruption following Intracerebral Hemorrhage in Rats. Journal of Biosciences and Medicines, 9, 125-137.

https://doi.org/10.4236/jbm.2021.99011

Received: July 23, 2021

Accepted: September 15, 2021

Published: September 18, 2021

Copyright ( 2021 by author(s) and Scientific Research Publishing Inc. This work is licensed under the Creative Commons Attribution International License (CC BY 4.0). http://creativecommons.org/licenses/by/4.0/

\section{Open Access}

\begin{abstract}
Mesenchymal Stem Cells (MSCs) are a type of non-hematopoietic progenitor cells which have self-replication capacity and multilineage differentiation. They have widely applied in studies of various diseases due to their effects in damaged tissue repair, neuroprotection and immunoregulation. MSCs can secret exosomes through multiple ways in the physiological or pathological state. Many researches' results on MSC-Exo show that it possesses many functions similar to MSCs, such as immunoregulation and regeneration promotion of damaged tissues. Hence, MSC-Exo is believed to have considerable research potentials in regenerative medicines. This study reviewed the research progresses on biological characteristics and functions of MSC-Exo.
\end{abstract}

\section{Keywords}

Bone Mesenchymal Stem Cell-Derived Exosome, Blood-Brain Barrier, Intracerebral Hemorrhage

\section{Introduction}

Mesenchymal Stem Cells (MSCs) are a type of non-hematopoietic progenitor cells which have self-replication capacity and multilineage differentiation. They have extensive distributions in bone marrow [1] [2], fat, liver, spleen, thymus, umbilical cord blood, placenta, Wharton's jelly, brain, lungs and dental pulps ${ }^{*}$ Gang Yang and Zhanwei Ruan contributed equally to this paper.

"Corresponding author. 
[3]-[7]. They have widely been applied in studies of various diseases due to their effects in damaged tissue repair, neuroprotection and immunoregulation. Previous research opinions agree that stem cells repair damaged tissues mainly through homing differentiation. In 2010, Lai et al. [8] separated Mesenchymal Stem Cells derived Exosomes (MSC-Exo) for the first time. Subsequent studies have proved the important biological functions of MSC-Exo [9]. MSC-Exo is a membranous vesicle which is secreted by MSCs and can carry and deliver bioactive substances (e.g. endogenous proteins, lipids, mRNA, siRNA, rRNA and micro RNA (miRNA)) which are wrapped in the lipid bilayers through internalization of target cells, receptor-ligand interaction or plasma membrane fusion. MSC-Exo is not only an important media for communicating among cells [10] [11], but also participates in maintaining the homeostasis of tissues. MSCs can secret exosomes through multiple ways in the physiological or pathological state. Some study has pointed that MSCs are the cells which secrete exosomes the mostly [9]. May research results on MSC-Exo show that it possesses many functions similar with MSCs, such as immunoregulation and regeneration promotion of damaged tissue. Hence, MSC-Exo is believed to have considerable research potentials in regenerative medicines.

Exosomes which are secreted by Neural Stem Cells (NSCs) can strengthen neuroplasticity and promote nerve regeneration and functional recovery after injury. They provide a therapeutic effect by regulating the functions of neurons and gliocytes in the local microenvironment and remote target cells [12]. Therefore, NSCs-Exo has remarkable clinical application potentials and they are a new potential treatment for nervous system disease. This study reviewed the research progresses on biological characteristics and functions of MSC-Exo.

\section{Biological Characteristics of MSC-Exo}

\subsection{Basic Features of MSC-Exo}

Exosomes refer to vesicles (diameter: $40-100 \mathrm{~nm}$ ) which are secreted by cells under different stress states and they are derived from the endosome system of cells. Firstly, cells sink through endocytosis and form the early endosome. Subsequently, the endosome membranes sink again to form intra-cavity vesicles which selectively receive proteins and lipids from the cytoplasm to form the late endosome. Next, the late endosome membrane and cytomembrane integrate to release a lot of vesicles out of cells through exocytosis, which are exosomes. The process for MSCs secretion of exosomes is the same with the process of other cells, which is also a process of "invagination-fusion-exsecretion" [13] [14]. MSCExo is wrapped by lipid bilayer and presents as cup-like minutes under an electron microscope. They can enrich in the $1.10-1.19 \mathrm{~g} / \mathrm{ml}$ sucrose density gradient solution through the 100,000 g ultracentrifugation [15]. The MSC-Exo surface contain membrane-related proteins (e.g. CD9, CD63, CD81 andCD83, MHC-I and MHC-II), heat shock proteins (Hspa8, Hsp70 and Hsp90), Guanosine Triphosphate (GTP), and multivesicular biogenic marker proteins (Alix and 
TSG101) [15] [16] [17], as well as metabolic enzymes (e.g. GAP-DH, LDHA, PGK1, aldolase and PKM), cytoskeletal proteins (actin, moesin and syntenin) as well as carrier proteins (e.g. albumin). Besides, MSC-Exo also expresses relevant molecules of MSCs, such as CD29, CD44, CD90 and CD73 [18]. In addition to proteins, MSC-Exo also is enrich of multiple biomacromolecules related with its biological source, including lipidosomes (e.g. phosphoglyceride, lysophosphatidic acid and cholesterol), ceramide, fatty acid chains, mRNA, non-coding RNA (miRNAs, tRNAs, rRNAs), and so on [19] [20] [21]. Exosomes is derived from the combination of vesicles and cytomembrane. Hence, secretion of exosomes is sensitive to different physicochemical substances and environmental conditions. For example, gamma knife-irradiation, calcium ion proteins, heparinase, statins, hypoxia and acidosis all can increase secretion of exosomes [19]. The hypoxia condition in placenta MSC media, depolarization of potassium ion dependent nerve cells and T-cell activation caused by TCR/CD3 crosslinking all can induce abundant secretion of exosomes [22].

\subsection{Unique microRNA in MSC-Exo}

Nucleic acids in MSC-Exo mainly include mRNA, miRNA, DNA, and so on [23]. MSCs can generate biological effect with target cells through miRNA in MSC-Exo [24]. Ti et al. [25] analyzed the miRNA expression spectra of MSC-Exo based on the gene chip technology and identified 42 miRNAs with abnormal expressions, including 15 upregulated. Specifically, expressions of miR-21, miR-146a and miR-181 are upregulated the mostly. According to the big data analysis, miR-21, miR-146a and miR-181 not only participate in cell development, congenital immunioreaction, inflammatory reaction toll-like receptor (TLR) pathway and IL-6 mediated cell signaling pathway [26], but also participate in the immune system activation and inflammation occurrence in the process of damaged tissue repair. miRNAs in MSC-Exo exchanges biological information among adjacent cells as a molecular switch and its content is changed significantly [27]. Microenvironmental factors, such as different cell sources as well as different calcium ions and growth factor concentration, all can influence miRNAs composition in exosomes, thus influencing the information transmission and regulation performance [28]. Montecalvo et al. [29] pointed out that miRNA in exosomes has high stability because of their unique membrane structure. They can resistant strong environmental interferences, such as low-acid $\mathrm{pH}$ value, low-temperature storage and RNAse-mediated degradation reaction. So far, abnormal expressions of exosomes-derived miRNA have been detected in many diseases. Therefore, miRNA in exosomes is viewed as the biomarker in many pathogenetic processes [30].

\subsection{Separation, Identification and Storage of MSC-Exo}

It usually uses supercentrifugation combined with sucrose density gradient to separate and purify exosomes from supernate of MSC media and from different 
biological fluids. It can eliminate solid precipitates of cells and big particles and separate exosomes under the ultra-high centrifugal force of 100,000 g [31]. In addition, High Performance Liquid Chromatography (HPLC), ultrafiltration method, antibody capture method and exosomes separation kit based on lectin (e.g. Exo Quick) are also good choices [32]. Some study believes that low-pH is conducive to separate exosomes and increase output [33]. There are many methods to identify exosomes, including Atomic Force Microscope (AFM), Scanning Electron Microscope (SEM), transmission electron microscope (TEM), Dynamic Light Scattering (DLS), Flow Cytometry (FCM), Nanoparticle Tracking Analysis (NTA), western blotting or Elisa method [32]. It usually suggested that the separated MSC-Exo shall be detected or frozen under a low temperature as soon as possible. Konala et al. [34] studied stability of exosomes under different storage conditions of $-20^{\circ} \mathrm{C}, 4^{\circ} \mathrm{C}$ and $37^{\circ} \mathrm{C}$, and found that exosomes under $4^{\circ} \mathrm{C}$ and $37^{\circ} \mathrm{C}$ shrank in volume, with some structural changes. Sokolova et al. [35] discovered that exosomes which were gained through ultracentrifugation can be stored under $-20^{\circ} \mathrm{C}$ for 6 months withoout any cryoprotectant. The size and structure have no significant changes even after many freezing $\left(-20^{\circ} \mathrm{C}\right)$-thawing cycles.

\section{Characteristics and Applications of NSCs}

NSCs are primary progenitor cells of nervous system in different development stages. They can renew by themselves and differentiated to neurons, astrocytes and oligodendrocytes. Therefore, NSCs play a vital role in the formation of central nervous system. NSCs which are separated from brain tissues or NSCs after pluripotent stem cell induced amplification are used to treat cerebral nerve injury [36] [37] [38] [39]. This mainly develops the role by mediating nerve regeneration and plasticity and weakening neuroinflammation [40] [41] [42]. Although NSCs transplantation therapy has extremely promising application prospects, their clinical applications are restricted by cell dedifferentiation, immunological rejection, stem cell tumorigenesis and low survival rate of stem cells. As a result, the substitutive therapy of NSCs-Exo attracts considerable attentions, which can be used to nerve injury and neurodegenerative disorders.

NSCs-Exo and other cells are mainly gained through 5 pathways: 1) supercentrifugation, including differential centrifugation, density gradient centrifugation combining with saccharose or iodixanol; 2) ultrafiltration and size exclusion chromatography; 3 ) polymer precipitation method using hydrophilic polymer, such as polyethylene glycol; 4) immunoaffinity capture chromatography; 5) microcontroller chip separation method [43] [44]. Currently, researchers usually adopt supercentrifugation.

According to cliniopathologic analysis of many neurodegenerative disorders, exosomes can serve as media of disease development. It is reported that exosomes secreted by nerve cells mediate the development of diseases by delivering specific pathogenic particles, such as $\beta$-amyloid peptide and tau proteins in Alz- 
heimer disease [45] [46] and $\alpha$-synuclein in Parkinson's disease [47], from the primitive cells to other cells. On the other hand, exosomes also have some neuroprotective effect, promote peripheral nerve regeneration and repair nerve damages. Besides, NSCs-Exo carries and delivers bioactive RNA, proteins and lipids. They can not only participate in synaptic transmission and neuron depolarization [48] as well as information exchange between neurons and neurogliocyte [49], but also regulate the synapsis function and maintain neurovascular integrity and myelinogenesis [50]. Under this background, exosomes which area released by NSCs have attracted wide attentions.

NSCs-Exo has the following functions: 1) mediating biological effect. They regulate cell growth and apoptosis through the specific miRNA, having some neuroprotection. Based on sequencing, Stevanato et al. [51] found 113 miRNAs in exosomes which are secreted by human NSCs. Results show that hsa-miR-1246, hsa-miR-4488, hsa-miR-4508, hsa-miR-4492 and hsa-miR-4516 have relatively high expression levels and hsa-miR-1246 is a target miRNA [52] that plays an important role in regulating cell growth and apoptosis [53]. In addition, other studies have pointed out that the high expressions of miR-21a and miR-125b in NSCs-Exo can promote differentiation and regeneration of nerve cells [54] [55]; 2) Mediating communication between NSCs and microenvironment. Cossetti et al. [56] found in a study that exosomes mediate communication between NSCs in mice and microenvironment and proinflammatory factor activate gamma interferon signal transduction pathway in NSCs, so that specific components of the gamma interferon pathway act on the target cells through exosomes [56]; 3) Mediating virus invasion into cells through non-receptor modes. For example, exosomes which are excreted by NSCs are added in the coxsackie virus and Adenovirus Receptor (CAR) defect cells and Adenovirus type 5 (AD5) for co-culture, which can promote invasion of Adenovirus type 5 into the coxsackie virus and Adenovirus Receptor defect cells [57]; 4) Playing a role as independent metabolic units. Exosomes can change concentration of key nutrients in the microenvironment, thus influencing physiological functions of peripheral cells [18]; 5) Influencing the aging process. It is found in a study that hypothalamus NSCs in mice influence the aging process after birth by releasing exosomes which carry specific miRNA into Cerebrospinal Fluid [58]; 6) Influencing morphological formation of microglial cells. According to the latest study, NSCs-Exo in the subventricular zone of mice is rich in miR-9, miR-26 and miR-181. These miRNAs specific target microglial cells influence the morphology, functions and proliferation in nervous system after birth [59] [60]. Therefore, NSCs-Exo has considerable promising application prospects to treat nervous system disorders.

The NSCs-Exo is superior to NSCs in promoting tissue regeneration and repair [61]. Therefore, NSCs-Exo has been widely used to study treatment for multiple nerve diseases. Compared with NSCs transplantation therapy, treatment based on NSCs-Exo has following advantages: 1) exosomes can be delivered to different regions of brain through nasal inhalation [62]; 2) Exosomes 
disassemble quickly after releasing drugs since they cannot duplicate in vivo. Hence, it is hardly to develop tumor cancerization after treatment with exosomes [63]; 3) Exosomes are vesicles which have extremely low possibility of small vascular obstruction after intravenous injection and penetrate the blood-brain barrier (BBB) [64] [65]; 4) Relatively low immunogenicity [65]. Obviously, it is feasible to use exosomes which are produced by large cell factories to treat diseases [66]. NSCs-Exo provides a cell-free therapy for nervous system disorders, without evident side-effects.

\section{Applications of Exosomes in Cerebral Stroke Treatment}

Stroke is one of major causes of deaths and disability. However, there's no drug that can promote neural functional recovery on the market yet. For most survivors from stroke, it takes a long rehabilitation process and long-term drug therapy. Hence, it is necessary to develop a substitutive therapy that can repair cerebral damages of patients with stroke. WEBB et al. [67] found that intravenous injection of NSCs-Exo into mice after thromboembolic stroke could promote repair of damaged cells, tissues and functions. NSCs-Exo promotes polarization of microglial cell into M2 phenotype, thus promoting clearance of debris and decrease chronic inflammation [68] [69]. Hence, NSCs-Exo can provide neuroprotection, relieve movement and memory disorder. Subsequently, WEBB et al. [70] continued to explore the therapeutic effect of NSCs-Exo in the pig stroke model. After treatment with intravenous injection of exosomes, they found that damaged tissues and functions of the pig stroke model are improved significantly, and the Alba integrity is maintained.

Xin et al. [71] found that intravenous infusion of NSCs-exosomes to patients with cerebral stroke can promote neurogenesis, neurite regeneration and angiogenesis. MSC-Exo can provide neuroprotective effect through multiple ways since it can penetrate through $\mathrm{BBB}$, while most drugs cannot [72]. Zhang et al. [73] provided two-week treatment of MSC-Exo to rats in the middle cerebral artery occlusion model and found that neurological functions of the model animals were improved significantly and the modified neurological severity scores (mNss) was increased to some extent. After four-week treatment of MSC-Exo, the quantity of axons surrounding the Ischemic Boundary zone (IBz) was increased significantly and nerve regeneration signals could be detection/This proved that MSC-Exo could induce axon development [72]. Besides, some study found that MSC-Exo contains encephalin with high activity and can decrease the newly synthesized $\beta$-amyloid beta protein content in cells after it invades into nerve cells [73]. Hence, it shows potentials in treatment of Alzheimer disease. These functions of MSC-Exo provide a new possibility to treat the neurodegenerative diseases.

$\mathrm{BBB}$ which has a high differential permeability strictly restricts the delivery of macromolecules and almost micromolecules from other organs to the brain [74]. With small volume and biocompatibility, exosomes can penetrate through BBB and has very high flexibility in treatment of central nervous system disorders. It can delivery proteins and RNA to brains through nasal inhalation, intravenous 
injection, abdominal injection and intracranial injection [74] [75]. HANEY et al. [75] treated the Parkinson's disease in mice by nasal administration of catalase-loaded exosomes and results demonstrated that catalase-loaded exosomes weakened the neuritis of mice with Parkinson's disease as a "drug" and it had significant neuroprotective effect. Some study proved that intravenous injection of exosomes which carry with specific miRNA and siRNA can target at nerve cells in brain and deliver miRNA and siRNA to target cells to treat mice with Alzheimer's disease [75] [76]. Joshi [77] pointed out that NSCs-Exo can penetrate through $\mathrm{BBB}$ effectively as a biological nano-carrier. In a word, there are two methods applied to study the therapeutic effect of miRNA of exosomes in central nervous system disorders. One is direct nasal inhalation or injection of exosomes in which there are miRNA good for the treatment. The other can load miRNA which can regulate disease-related gene expressions or specific miRNA that can promote nerve regeneration into exosomes selectively, and then make an in vivo study.

To sum up, NSCs-Exo treatment for stroke has five characteristics. It has neuroprotection to improve behavior and activity, eliminate intracranial hemorrhage conversion of ischemic diseases, weaken cerebral injury volume and brain swelling, and promote functional recovery after injury. Although there are few studies on NSCs-Exo treatment for stroke, It still great potential.

\section{Conclusion}

Although there are few studies on MSC-Exo, it has shown strong potential in medical treatment and exciting therapeutic effects in some animal models. Exosomes are the ideal carrier of drugs or gene transmission. They can release mRNA and miRNA into cytoplasm directly through membrane fusion with target cells, or uptake bioactive substances through endocytosis of target cells. Alternatively, they can recognize specific receptors on the cell surface and regulate the microenvironment of the disease, thus changing the initiation and development of the disease. MSC-Exo is a relatively unique type of exosomes and has some characteristics of stem cells. These studies prove that MSC-Exo can repair damaged tissues, inhibit inflammatory responses and regulate the immune system. However, the influences of MSC-Exo on tumors are still controversial and have to be further studied. Compared with MSC, MSC-Exo has a smaller volume, higher structural stability, lower immunogenicity and zero infection risk [57] [58]. Hence, MSC-Exo is highly appreciated as a cell-free therapy in studies and clinical studies; it is expected to enter into clinical trial soon. However, the extraction process of secreted MSC-Exo is relatively complicated and the components wrapped by MSC-Exo are sensitive to the original cells and environmental conditions. For clinical use of MSC-Exo, the primary task might be to simplify and standardize the MSC-Exo extraction to get MSC-Exo with stable properties.

\section{Fund-Supported Projects}

Zhejiang Health Development Foundation 2019ZA127. 
Shaoxing Health Science and Technology Plan 2017CX025.

Zhejiang Health Development Foundation 2019ZD059.

Zhejiang Medical Association Clinical Research Fund Projects 2019ZYC-A157.

\section{Conflicts of Interest}

The authors declare no conflicts of interest regarding the publication of this paper.

\section{References}

[1] Gallina, C., Turinetto, V. and Giachino, C. (2015) A New Paradigm in Cardiacregeneration: The Mesenchymal Stem Cell Secretome. Stem Cells International, 2015, Article ID: 765846. https://doi.org/10.1155/2015/765846

[2] Kariminekoo, S., Movassaghpour, A., Rahimzadeh, A., Talebi, M., Shamsasenjan, K. and Akbarzadeh, A. (2016) Implications of Mesenchymal Stem Cells in Regenerative Medicine. Artificial Cells, Nanomedicine, and Biotechnology, 44, 749-757. https://doi.org/10.3109/21691401.2015.1129620

[3] Lopez-Verrilli, M.A., Caviedes, A., Cabrera, A., Sandoval, S., Wyneken, U. and Khoury, M. (2016) Mesenchymal Stem Cell-Derived Exosomes from Different Sources Selectively Promote Neuritic Outgrowth. Neuroscience, 320, 129-139. https://doi.org/10.1016/j.neuroscience.2016.01.061

[4] Lotfy, A., Salama, M., Zahran, F., Jones, E., Badawy, A. and Sobh, M. (2014) Characterization of Mesenchymal Stem Cells Derived from Rat Bone Marrow and Adipose Tissue: A Comparative Study. International Journal of Stem Cells, 7, 135-142. https://doi.org/10.15283/ijsc.2014.7.2.135

[5] De Miguel, M.P., Fuentes-Julian, S., Blazque-martinez, A., Pascual, C.Y., Aller, M.A., Arias, J., et al. (2012) Immunosuppressive Properties of Mesenchymal Stem Cells: Advances and Applications. Current Molecular Medicine, 12, 574-591. https://doi.org/10.2174/156652412800619950

[6] Lotfinegad, P., Shamsasenjan, K., Movassaghpour, A., Majidi, J. and Baradaran, B. (2014) Immunomodulatory Nature and Site Specific Affinity of Mesenchymal Stem Cells: A Hope in Cell Therapy. Advanced Pharmaceutical Bulletin, 4, 5-13. https://doi.org/10.5681\%2Fapb.2014.002

[7] Kellner, J., bivajothi, S. and Mcniece, I. (2015) Differential Properties of Human Stromal Cells from Bone Marrow, Adipose, Liver and Cardiac Tissucs. Cytotherapy, 17, 1514-1523. https://doi.org/10.1016/j.jcyt.2015.07.009

[8] Lai, R.C., Arslan, F., Lee, M.M., Sze, N.S.K., Choo, A., Chen, T.S., et al. (2010) Exosome Secreted by MSC Reduces Myocardial Ischemia/Reperfusion Injury. Stem Cell Research, 4, 214-222. https://doi.org/10.1016/j.scr.2009.12.003

[9] Yeo, R.W., Laj, R.C., Zhang, B., Tan, S.S., Yin, Y., The, B.J., et al. (2013) Mesenchymal Stem Cell: An Emcient Mass Producer of Exosomes for Drug Delivery. Advanced Drug Delivery Reviews, 65, 336-341. https://doi.org/10.1016/j.addr.2012.07.001

[10] Ailawadi, S., Wang, X., Gu, H. and Fan, G.-C. (2015) Pathologic Function and Therapeutic Potential of Exosomes in Cardiovascular Disease. Biochimica et Biophysica Acta (BBA)-Molecular Basis of Disease, 1852, 1-11.

https://doi.org/10.1016/j.bbadis.2014.10.008

[11] Huang, L., Ma, W., Ma, Y., Feng, D., Chen, H. and Cai, B. (2015) Exosomes in Mesenchymal Stem Cells, a New Therapeutic Strategy for Cardiovascular Diseases. In- 
ternational Journal of Biological Sciences, 11, 238-245.

[12] Bai, Y., Han, Y.D., Yan, X.L., Ren, J., Zeng, Q., Li, X.-D., et al. (2018) Adipose Mesenchymal Stem Cell-Derived Exosomes Stimulated by Hydrogen Peroxide Enhanced Skin Flap Recovery in Ischemia-Reperfusion Injury. Biochemical and Biophysical Research Communications, 500, 310-317. https://doi.org/10.1016/j.bbrc.2018.04.065

[13] Simons, M. and Raposo, G. (2009) Exosomes-Vesicular Carreers for Intercelllar Communication. Current Opinion in Cell Biology, 21, 575-581. https://doi.org/10.1016/j.ceb.2009.03.007

[14] Huotri, J. and Helenius, A. (2011) Endosome Maturation. The EMBO Journal, 30, 3481-3500. https://doi.org/10.1038/emboj.2011.286

[15] Mathivanan, S., Ji, H. and Simpson, R.J. (2010) Exosomes: Extracellular Organelles Important in Intercellular Communication. Journal of Proteomics, 73, 1907-1920. https://doi.org/10.1016/j.jprot.2010.06.006

[16] Hannafon, B.N. and Ding, W.Q. (2013) Intercellular Communication by Exosome-Derived MicroRNAs in Cancer. International Journal of Molecular Sciences, 14, 14240 14269. https://doi.org/10.3390/ijms140714240

[17] Zeringer, E., Barta, T., Li, M. and Vlassov, A.V. (2015) Strategies for Isolation of Exosomes. Cold Spring Harbor Protocols, 2015, 319-323.

https://doi.org/10.1101/pdb.top074476

[18] Wang, R., Lin, S., Li, L., et al. (2014) Protective Effect of Bone Marrow Mesenchymal Stem Cell Source Exosome on Renal Ischemia-Reperfusion Injury in Rats. Chinese Medical Journal, 94, 3298-3303.

[19] Subra, C., Laulagnier, K., Perret, B. and Record, M. (2007) Exosome Lipidomics Unravels Lipidsorting at the Level of Multivescular Bodies. Biochimie, 89, 205-212. https://doi.org/10.1016/j.biochi.2006.10.014

[20] Daviran, M., Catalano, J. and Schultz, K.M. (2020) Determining How Human Mesenchymal Stem Cells Change Their Degradation Strategy in Response to Microenvironmental Stiffness. Biomacromolecules, 21, 3056-3068. https://doi.org/10.1021/acs.biomac.0c00432

[21] Yoon, Y.J., Kim, O.Y. and Gho, Y.S. (2014) Extracellular Vesicles as Emerging Intercellular Communicasomes. BMB Report, 47, 531-539.

https://doi.org/10.5483/BMBRep.2014.47.10.164

[22] Salomon, C., Ryan, J., Sobrevia, L., Kobayashi, M., Ashman, K., Mitchell, M., et al. (2013) Exosomal Signaling during Hypoxia Mediates Microvascular Endothelial Cell Migration and Vasculogenesis. PLOS ONE, 8, e68451. https://doi.org/10.1371/journal.pone.0068451

[23] Chen, T.S., Lai, R.C., Lee, M.M., Choo, A.B.H., Lee, C.N. and Lim, S.K. (2010) Mesenchymal Stem Cell Secretes Microparticles Enriched in Pre-MicroRNAs. Nucleic Acids Research, 38, 215-224. https://doi.org/10.1093/nar/gkp857

[24] Yu, B., Zhang, X. and Li, X. (2014) Exosomes Derived from Mesenchymal Stem Cells. International Journal of Molecular Sciences, 15, 4142-4157. https://doi.org/10.3390/ijms15034142

[25] Ti, D., Hao, H., Tong, C., Liu, J., Dong, L., Zheng, J., et al. (2015) LPS-Preconditi Mesenchymal Stromal Cells Modify Macrophage Polarization for Resolution of Chronic Inflammation via Exosome-Shuttled Let-7b. Journal of Translational Medicine, 13, Article No. 308. https://doi.org/10.1186/s12967-015-0642-6

[26] Navakanitworakul, R., Hung, W.T., Gunewardena, S., Davis, J.S., Chotigeat, W. and Christenson, L.K. (2016) Characterization and Small RNA Content of Extracellular Vesicles in Follicular Fluid of Developing Bovine Antral Follicles. Scientific Reports, 
6, Article No. 25486. https://doi.org/10.1038/srep25486

[27] Hunter, M.P., Ismail, N., Zhang, X., Aguda, B.D., Lee, E.J., et al. (2008) Detection of MicroRNA Expression in Human Peripheral Blood Microvesicles. PLoS ONE, 3, e3694. https://doi.org/10.1371/journal.pone.0003694

[28] Montecalvo, A., Larregina, A.T., Shufesky, W.J., Stolz, D.B., Sullivan, M.L.G., Karlsson, J.M., et al. (2012) Mechanism of Transfer of Functional MicroRNAs between Mouse Dendritic Cells via Exosomes. Blood, 119, 756-766.

https://doi.org/10.1182/blood-2011-02-338004

[29] Taverna, S., Fontana, S., Monteleone, F., Pucci, M., Saieva, L., De Caro, V., et al. (2016) Curcumin Modulates Chronic Myelogenous Leukemia Exosomes Composition and Affects Angiogenic Phenotype, via Exosomal miR-21. Oncotarget, 7, 3042030439. https://doi.org/10.18632/oncotarget.8483

[30] Thery, C., Amigorena, S., Raposo, G. and Clayton, A. (2006) Isolation and Characterization of Exosomes from Cell Culture Supernatants and Biologica Fluids. Current Protocols in Cell Biology, 30, 3.22.1-3.22.29. https://doi.org/10.1002/0471143030.cb0322s30

[31] Cheng, L. and Xu, T. (2015) Development of Mesenchymal Stem Cell Derived Exosomes and Its Research Progress in Tumor Therapy. Modern Oncology Medicine, 24, 2470-2473.

[32] Cao, F., Gao, Y., Chu, Q., et al. (2019) Proteomics Comparison of Exosomes from Serum and Plasma between Ultracentrifugation and Polymer-Based Precipitation Kit Methods. Electrophoresis, 40, 3092-3098. https://doi.org/10.1002/elps.201900295

[33] Ban, J.J., Lee, M., Im, W. and Kim, M. (2015) Low pH Increases the Yield of Exosome Isolation. Biochemical and Biophysical Research Communications, 461, 76-79. https://doi.org/10.1016/j.bbrc.2015.03.172

[34] Konala, V.B., Mamidi, M.K., Bhonde, R., Das, A.K., Pochampally, R. and Pal, R. (2016) The Current Landscape of the Mesenchymal Stromal Cell Secretome: A New Paradigm for Cell-Free Regeneration. Cytotherapy, 18, 13-24. https://doi.org/10.1016/j.jcyt.2015.10.008

[35] Sokolova, V., Ludwig, A.K., Hornung, S., Rotan, O., Horn, P.A., Epple, M., et al. (2011) Characterisation of Exosomes Derived from Human Cells by Nanopartice Tracking Analysis and Scanning Elctron Microscopy. Colloids Surf B: Biointerlaces, 87, 146-150. https://doi.org/10.1016/j.colsurfb.2011.05.013

[36] Kriegstein, A. and Alvarez-Buylla, A. (2009) The Glial Nature of Embryonic and Adult Neural Stem Cells. Annual Review of Neuroscience, 32, 149-184.

https://doi.org/10.1146/annurev.neuro.051508.135600

[37] Hattiangady, B. and Shetty, A.K. (2012) Neural Stem Cell Grafting Counteracts Hippocampal Injury-Mediated Impairments in Mood, Memory, and Neurogenesis. Stem Cells Translational Medicine, 1, 696-708. https://doi.org/10.5966/sctm.2012-0050

[38] Goldman, S.A. (2016) Stem and Progenitor Cell-Based Therapy of the Central Nervous System: Hopes, Hype, and Wishful Thinking. Cell Stem Cell, 18, 174-188. https://doi.org/10.1016/j.stem.2016.01.012

[39] Shetty, A.K. (2014) Hippocampal Injury-Induced Cognitive and Mood Dysfunction, Altered Neurogenesis, and Epilepsy: Can Early Neural Stem Cell Grafting Intervention Provide Protection. Epilepsy \& Behavior, 38, 117-124.

https://doi.org/10.1016/j.yebeh.2013.12.001

[40] Kokaia, Z. and Lindvall, O. (2018) Sensors of Succinate: Neural Stem Cell Grafts Fight Neuroinflammation. Cell Stem Cell, 22, 283-285.

https://doi.org/10.1016/j.stem.2018.01.019 
[41] Peruzzotti-Jametti, L., Bernstock, J.D., Vicario, N., Costa, A.S.H., Kwok, C.K., Leonardi, T., et al. (2018) Macrophage-Derived Extracellular Succinate Licenses Neural Stem Cells to Suppress Chronic Neuroinflammation. Cell Stem Cell, 22, 355-368.e13. https://doi.org/10.1016/j.stem.2018.01.020

[42] Cheng, Z., Zhu, W., Cao, K., Wu, F., Li, J., Wang, G., et al. (2016) Anti-Inflammatory Mechanism of Neural Stem Cell Transplantation in Spinal Cord Injury. International Journal of Molecular Sciences, 17, Article No. 1380. https://doi.org/10.3390/ijms17091380

[43] Li, P., Kaslan, M., Lee, S.H., Yao, J. and Gao, Z. (2017) Progress in Exosome Isolation Techniques. Theranostics, 7, 789-804. https://doi.org/10.7150/thno.18133

[44] Yang, X.X., Sun, C., Wang, L. and Guo, X.-L. (2019) New Insight into Isolation, Identification Techniques and Medical Applications of Exosomes. Journal of Controlled Release, 308, 119-129. https://doi.org/10.1016/j.jconrel.2019.07.021

[45] Rajendran, L., Honsho, M., Zahn, T.R., Keller, P., Geiger, K.D., Verkade, P., et al. (2006) Alzheimer's Disease Beta-Amyloid Peptides Are Released in Association with Exosomes. Proceedings of the National Academy of Sciences of the United States of America, 103, 11172-11177. https://doi.org/10.1073/pnas.0603838103

[46] Asai, H., Ikezu, S., Tsunoda, S., Medalla, M., Luebke, J., Haydar, T., et al. (2015) Depletion of Microglia and Inhibition of Exosome Synthesis Halt Tau Propagation. Nature Neuroscience, 18, 1584-1593. https://doi.org/10.1038/nn.4132

[47] Emmanouilidou, E., Melachroinou, K., Roumeliotis, T., Garbis, S.D., Ntzouni, M., Margaritis, L.H., et al. (2010) Cell-Produced a-Synuclein Is Secreted in a Calciumdependent Manner by Exosomes and Impacts Neuronal Survival. Journal of Neuroscience, 30, 6838-6851. https://doi.org/10.1523/JNEUROSCI.5699-09.2010

[48] Lachenal, G., Pernet-Gallay, K., Chivet, M., Hemming, F.J., Belly, A., Bodon, G., et al. (2011) Release of Exosomes from Differentiated Neurons and Its Regulation by Synaptic Glutamatergic Activity. Molecular and Cellular Neuroscience, 46, 409-418. https://doi.org/10.1016/j.mcn.2010.11.004

[49] Paolicelli, R.C., Bergamini, G. and Rajendran, L. (2019) Cell-Tocell Communication by Extracellular Vesicles: Focus on Microglia. Neuroscience, 405, 148-157. https://doi.org/10.1016/j.neuroscience.2018.04.003

[50] Holm, M.M., Kaiser, J. and Schwab, M.E. (2018) Extracellular Vesicles: Multimodal Envoys in Neural Maintenance and Repair. Trends in Neurosciences, 41, 360-372. https://doi.org/10.1016/j.tins.2018.03.006

[51] Stevanato, L., Thanabalasundaram, L., Vysokov, N. and Sinden, J.D. (2016) Investigation of Content, Stoichiometry and Transfer of miRNA from Human Neural Stem Cell Line Derived Exosomes. PLoS ONE, 11, e0146353.

https://doi.org/10.1371/journal.pone.0146353

[52] Zhang, Y., Liao, J.M., Zeng, S.X. and Lu, H. (2011) p53 Downregulates Down Syndrome-Associated DYRK1A through miR-1246. EMBO Report, 12, 811-817. https://doi.org/10.1038/embor.2011.98

[53] Xu, L.J., Jiang, T., Zhao, W., Han, J.-F., Liu, J., Deng, Y.-Q., et al. (2014) Parallel mRNA and microRNA Profiling of HEV71-Infected Human Neuroblastoma Cells Reveal the Up-Regulation of miR-1246 in Association with DLG3 Repression. PLoS ONE, 9, e95272. https://doi.org/10.1371/journal.pone.0095272

[54] Ma, Y., Li, C., Huang, Y., Wang, Y., Xia, X. and Zheng, J.C. (2019) Exosomes Released from Neural Progenitor Cells and Induced Neural Progenitor Cells Regulate Neurogenesis through miR-21a. Cell Communication and Signaling, 17, Article No. 96. https://doi.org/10.1186/s12964-019-0418-3

[55] Takeda, Y.S. and Xu, Q. (2015) Neuronal Differentiation of Human Mesenchymal 
Stem Cells Using Exosomes Derived from Differentiating Neuronal Cells. PLoS $O N E, 10$, e0135111. https://doi.org/10.1371/journal.pone.0135111

[56] Cossetti, C., Iraci, N., Mercer, T.R., Leonardi, T., Alpi, E., Drago, D., et al. (2014) Extracellular Vesicles from Neural Stem Cells Transfer IFN-Gamma via Ifngr1 to Activate Stat1 Signaling in Target Cells. Molecular Cell, 56, 193-204. https://doi.org/10.1016/j.molcel.2014.08.020

[57] Sims, B., Gu, L., Krendelchtchikov, A. and Matthews, Q. (2014) Neural Stem Cell-Derived Exosomes Mediate Viral Entry. International Journal of Nanomedicine, 9, 4893-4897. https://doi.org/10.2147/IJN.S70999

[58] Zhang, Y., Kim, M.S., Jia, B., Yan, J., Zuniga-Hertz, J.P., Han, C., et al. (2017) Hypothalamic Stem Cells Control Ageing Speed Partly through Exosomal miRNAs. Nature, 548, 52-57. https://doi.org/10.1038/nature23282

[59] Morton, M.C., Neckles, V.N., Seluzicki, C.M., Holmberg, J.C. and Feliciano, D.M. (2018) Neonatal Subventricular Zone Neural Stem Cells Release Extracellular Vesicles That Act as a Microglial Morphogen. Cell Reports, 23, 78-89. https://doi.org/10.1016/j.celrep.2018.03.037

[60] Cunningham, C.L., Martinez-Cerdeno, V. and Noctor, S.C. (2013) Microglia Regulate the Number of Neural Precursor Cells in the Developing Cerebral Cortex. Journal of Neuroscience, 33, 4216-4233. https://doi.org/10.1523/JNEUROSCI.3441-12.2013

[61] Teixeira, F.G., Carvalho, M.M., Sousa, N. and Salgado, A.J. (2013) Mesenchymal Stem Cells Secretome: A New Paradigm for Central Nervous System Regeneration? Cellular and Molecular Life Sciences, 70, 3871-3882. https://doi.org/10.1007/s00018-013-1290-8

[62] Long, Q., Upadhya, D., Hattiangady, B., Kim, D.-K., An, S.Y., Shuai, B., et al. (2017) Intranasal MSC-Derived A1-Exosomes Ease Inflammation, and Prevent Abnormal Neurogenesis and Memory Dysfunction after Status Epilepticus. Proceedings of the National Academy of Sciences of the United States of America, 114, E3536-e3545. https://doi.org/10.1073/pnas.1703920114

[63] Mcculloh, C.J., Olson, J.K., Wang, Y., Zhou, Y., Tengberg, N.H., Deshpande, S., et al. (2018) Treatment of Experimental Necrotizing Enterocolitis with Stem Cell-Derived Exosomes. Journal of Pediatric Surgery, 53, 1215-1220. https://doi.org/10.1016/j.jpedsurg.2018.02.086

[64] Zhang, Z.G., Buller, B. and Chopp, M. (2019) Exosomes-Beyond Stem Cells for Restorative Therapy in Stroke and Neurological Injury. Nature Reviews Neurology, 15, 193-203. https://doi.org/10.1038/s41582-018-0126-4

[65] El Andaloussi, S., Lakhal, S., Mager, I. and Wood, M.J.A. (2013) Exosomes for Targeted siRNA Delivery across Biological Barriers. Advanced Drug Delivery Reviews, 65, 391-397. https://doi.org/10.1016/j.addr.2012.08.008

[66] Gimona, M., Pachler, K., Laner-Plamberger, S., Schallmoser, K. and Rohde, E. (2017) Manufacturing of Human Extracellular Vesicle-Based Therapeutics for Clinical Use. International Journal of Molecular Sciences, 18, Article No. 1190. https://doi.org/10.3390/ijms18061190

[67] Webb, R.L., Kaiser, E.E., Scoville, S.L., Thompson, T.A., Fatima, S., Pandya, C., et al. (2018) Human Neural Stem Cell Extracellular Vesicles Improve Tissue and Functional Recovery in the Murine Thromboembolic Stroke Model. Translational Stroke Research, 9, 530-539. https://doi.org/10.1007/s12975-017-0599-2

[68] Chen, J., Venkat, P., Zacharek, A. and Chopp, M. (2014) Neurorestorative Therapy for Stroke. Frontiers in Human Neuroscience, 8, Article No. 382. https://doi.org/10.3389/fnhum.2014.00382

[69] Gronberg, N.V., Johansen, F.F., Kristiansen, U. and Hasseldam, H. (2013) Leuko- 
cyte Infiltration in Experimental Stroke. Journal of Neuroinflammation, 10, Article No. 892. https://doi.org/10.1186/1742-2094-10-115

[70] Webb, R.L., Kaiser, E.E., Jurgielewicz, B.J., Spellicy, S., Scoville, S.L., Thompson, T.A., et al. (2018) Human Neural Stem Cell Extracellular Vesicles Improve Recovery in a Oorcine Model of Ischemic Stroke. Stroke, 49, 1248-1256.

https://doi.org/10.1161/STROKEAHA.117.020353

[71] Xin, H., Li, Y., Cui, Y., Yang, J.J., Zhang, Z.G. and Chopp, M. (2013) Systemic Administration of Exosomes Released from Mesenchymal Stromal Cells Promote Functional Recovery and Neurovascular Plasticity after Stroke in Rats. Journal of Cerebral Blood Flow \& Metabolism, 33, 1711-1715. https://doi.org/10.1038\%2Fjcbfm.2013.152

[72] Jarmalaviciute, A. and Pivoriunas, A. (2016) Exosomes as a Potential Novel Therapeutic Tools against Neurodegenerative Diseases. Pharmacological Research, 113, 816-822. https://doi.org/10.1016/j.phrs.2016.02.002

[73] Zhang, Y., Chopp, M., Liu, X.S., Katakowski, M., Wang, X., Tian, X., et al. (2016) Exosomes Derived from Mesenchymal Stromal Cells Promote Axonal Growth of Cortical Neurons. Molecular Neurobiology, 54, 2659-2673.

https://doi.org/10.1007/s12035-016-9851-0

[74] Almutairi, M.M., Gong, C., Xu, Y.G., Chang, Y. and Shi, H. (2016) Factors Controlling Permeability of the Blood-Brain Barrier. Cellular and Molecular Life Sciences, 73, 57-77. https://doi.org/10.1007/s00018-015-2050-8

[75] Haney, M.J., Klyachko, N.L., Zhao, Y., Gupta, R., Plotnikova, E.G., He, Z., et al. (2015) Exosomes as Drug Delivery Vehicles for Parkinson's Disease Therapy. Journal of Controlled Release, 207, 18-30. https://doi.org/10.1016/j.jconrel.2015.03.033

[76] Alvarez-Erviti, L., Seow, Y., Yin, H., Betts, C., Lakhal, S. and Wood, M.J.A. (2011) Delivery of siRNA to the Mouse Brain by Systemic Injection of Targeted Exosomes. Nature Biotechnology, 29, 341-345. https://doi.org/10.1038/nbt.1807

[77] Joshi, B.S. and Zuhorn, I.S. (2021) Heparan Sulfate Proteoglycan-Mediated Dynamin-Dependent Transport of Neural Stem Cell Exosomes in an In Vitro blood-Brain Barrier Model. European Journal of Neuroscience, 53, 706-719.

https://doi.org/10.1111/ejn.14974

\section{Abbreviations}

MSC-Exo: mesenchymal stem cell derived exosomes

BBB: blood brain barrier

MSCs: mesenchymal stem cells

NSCs: neural stem cells 